\title{
Monitoring Accreting X-ray Pulsars with the GLAST Burst Monitor
}

\author{
Colleen A. Wilson-Hodge, Mark H. Finger, Sandeep K. Patel, P.Narayana Bhat, \\ Robert D. Preece and Charles A. Meegan
}

National Space Science and Technology Center, Huntsville, AL 35805

\begin{abstract}
Accreting pulsars are exceptionally good laboratories for probing the detailed physics of accretion onto magnetic stars. While similar accretion flows also occur in other types of astrophysical systems, e.g. magnetic CVs, only neutron stars have a small enough moment of inertia for the accretion of angular momentum to result in measurable changes in spinfrequency in a timescale of days. Long-term monitoring of accreting pulsar spin-frequencies and fluxes was demonstrated with the Burst and Transient Source Experiment (BATSE) on the Compton Gamma Ray Observatory. Here we present sample results from BATSE, discuss measurement techniques appropriate for GBM, and estimate the expected GBM sensitivity.
\end{abstract}

Keywords: GLAST, Pulsars, X-ray binaries, accretion

PACS: $95.55 . \mathrm{Ka}, 97.60 . \mathrm{Gb}, 97.10 . \mathrm{Gz}, 97.60 . \mathrm{Gb}, 97.80 . \mathrm{Jp}$

\section{INTRODUCTION}

Accreting X-ray pulsars consist of a magnetized neutron star $\left(B=10^{12} \mathrm{G}\right)$ orbiting a companion. Accretion in these systems occurs via Roche lobe overflow, a wind, or interaction with the circumstellar disk of a Be star near periastron passage. Recently, Integral has discovered a number of likely highly absorbed wind accreting X-ray pulsars.

A large number of accreting pulsars were monitored daily with BATSE. For example, Figure 1 shows the spinfrequency history and the pulsed flux measured for the accreting Be/X-ray pulsar EXO 2030+375. Each point corresponds to a periastron passage in the system. Monitoring of this particular pulsar led to the discovery that the infrared magnitudes showed a decline shortly before the pulsar began to spin down and the pulsed flux abruptly dropped [2]. This implied that the density of the disk around the companion star dropped, making less material available for accretion onto the pulsar. Later, after BATSE was no longer able to observe, the infrared magnitudes again brightened accompanied by a brightening of the X-ray outbursts and a transition to spin-up [3].

\section{TECHIQUE}

The techniques used to detect known accreting pulsars with BATSE are described in detail elsewhere $[4,5]$. The basic idea is that the data were fitted with a segmented spline model for the background, continuous in value and first derivative across segment boundaries, but not across data gaps. For a particular pulsar, with an approximately known spin-frequency, pulse profiles were generated using a Fourier harmonic expansion in a pulse phase model that incorporated the known pulse frequency for short segments of data, short enough that the loss of power due to phase drift was small. These short segment profiles were then shifted using a grid in pulse frequency and sometimes frequency derivative. The shifted profiles were combined and the best frequency (and derivative) was selected from the grid using a modified Z-squared statistic. This technique should be easily adapted to GBM, building on the legacy of our BATSE work.

\section{ESTIMATED GBM SENSTIVITY}

We estimated the sensitivity $S$ of the GBM NaI detectors to accreting pulsars using

$$
S=N_{\text {sigma }}\left(\frac{A B}{T \Delta E A_{\mathrm{eff}}^{2}}\right)^{1 / 2}
$$



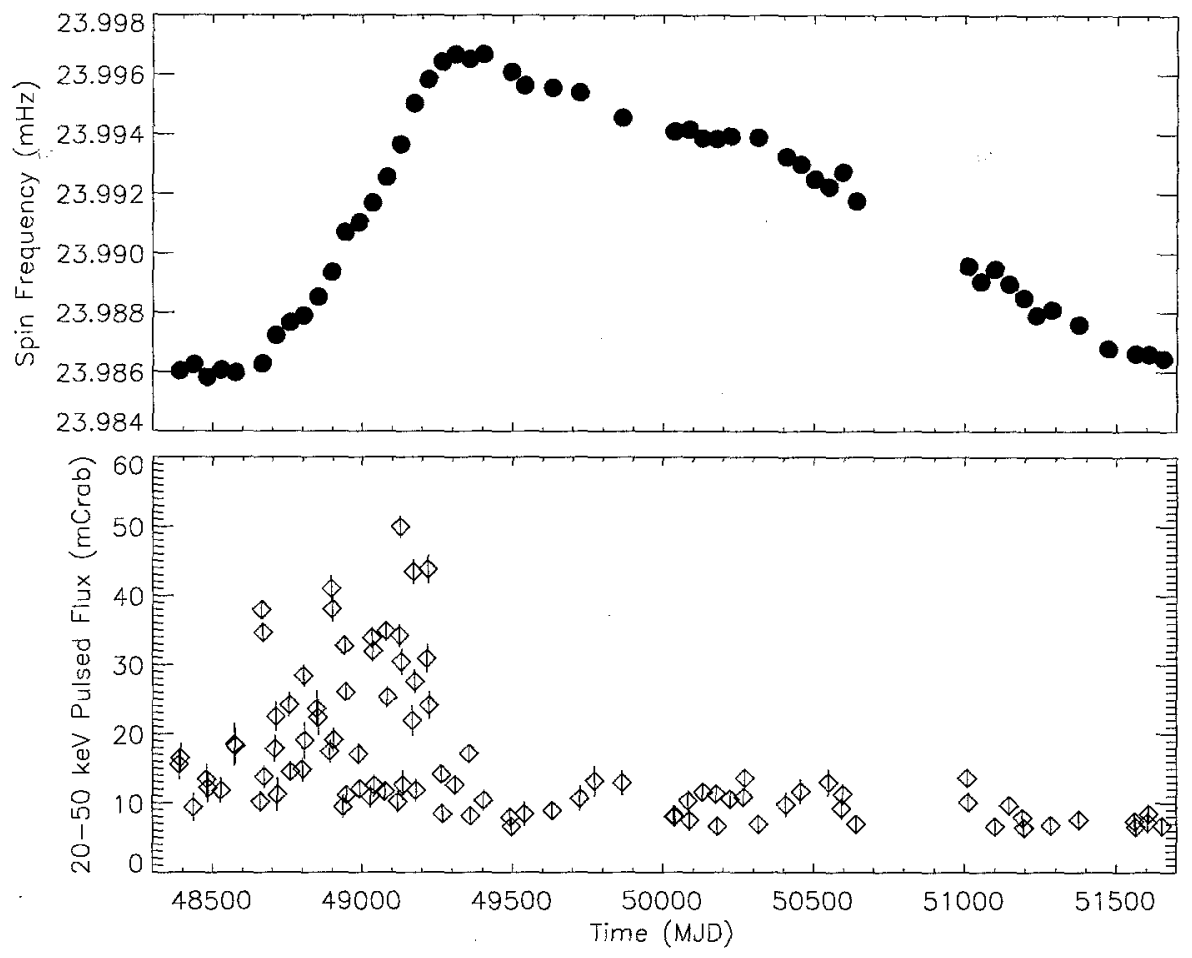

FIGURE 1. (top): Barycentered, orbit corrected spin-frequency for EXO $2030+375$ at each periastron passage where BATSE detected pulsations. (bottom): Corresponding $20-50 \mathrm{keV}$ pulsed flux for these observations measured with BATSE.

where $N_{\text {sigma }}=3$ is the significance, $A=252 \mathrm{~cm}^{2}$ is the geometric area for two NaI detectors, $B$ is the background rate per $\mathrm{cm}^{-2}, T=50000$ s is the estimated on-source time in one day, $\Delta E=E / 2$ is the energy bin width, and $A_{\text {eff }}$ is the effective area. The background rate was estimated by scaling BATSE SPEC detector measurements and correcting for the differing materials in front of the BATSE and GBM detectors. The effective area for two GBM NaI detectors was estimated from detector response matrices (DRMs) generated by Marc Kippen et al. at Los Alamos National Lab for two detectors at moderate angles for a particular pointing direction. These DRMs included spacecraft scattering but did not include atmospheric scattering.

Figure 2 shows the result of our sensitivity calculation. For reference, we have also done the same calculation for the BATSE Large area detectors (LADs) using actual measured background rates and actual DRMs for two detectors. GBM is considerably more sensitive than the BATSE LADs below about $25 \mathrm{keV}$ because the GBM detectors have only a $\mathrm{Be}$ window in front of them compared to the plastic scintillator, aluminum honeycomb, and aluminum window in front of the BATSE LADs. For reference, typical spectra of 4 accreting pulsars are shown that were detectable with BATSE and should be detectable with GBM.

\section{CONCLUSIONS}

We expect that GBM should be able to detect most of the X-ray pulsars observed with BATSE, despite GBMs smaller area. This is because of the fortunate combination of a large number of photons in the 8-25 keV range from most accreting pulsars combined with GBMs improved response over BATSE. This will be proposed as a Guest investigation. 


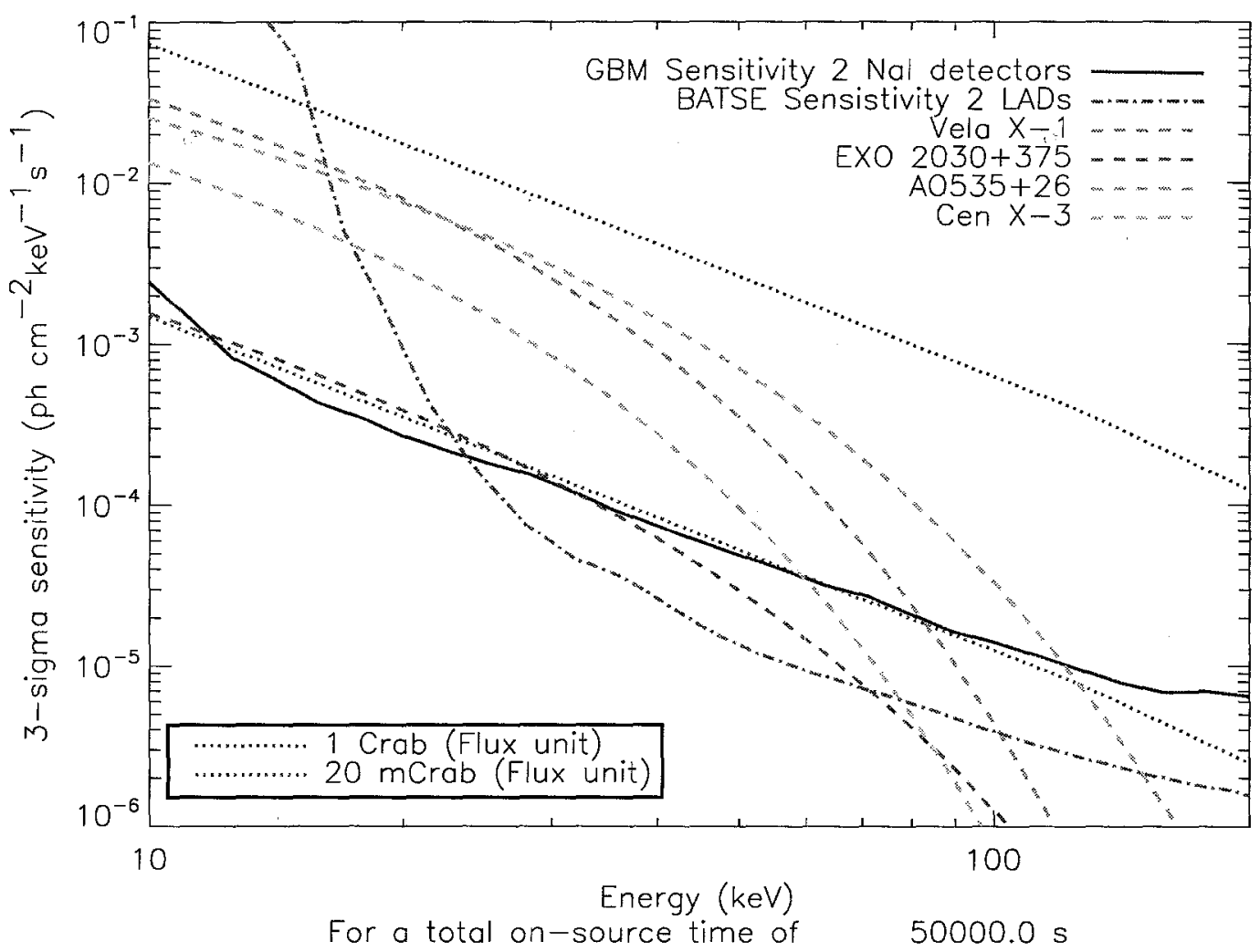

FIGURE 2. Estimated GBM 3- $\sigma$ sensitivity to accreting X-ray pulsars (blue line) for $50 \mathrm{ks}$ on-source. For comparison BATSE's sensitvity (pink dot-dash line) and representative spectra for the accreting X-ray pulsars Vela X-1 (light green), EXO $2030+375$ (dark green), A 0535+262 (light blue), and Cen X-3 (orange). One crab and 20 mCrab flux units are also shown for reference.

\section{ACKNOWLEDGMENTS}

The authors acknowledge Dr. Marc Kippen and his colleagues at LANL for providing the DRMs used in this paper.

\section{REFERENCES}

1. Walter, R. et al. $2006, A \& A, 453,133$

2. Wilson, C.A., Finger, M.H., Coe, M.J., Laycock, S., Fabregat, J. 2002, ApJ, 570, 287

3. Wilson, C.A., Fabregat, J., Coburn, W. 2005, ApJ, 620, L99

4. Bildsten, L., et al. 1997, ApJS, 113, 367

5. Finger, M.H., et al. 1999, ApJ, 517, 449 
glast_symp_abstract_psrs.txt

Monitoring Accreting $x$-ray Pulsars with the GLAST Burst Monitor

Colleen A. Wilson, Mark H. Finger, Sandeep K. Pate1, P. Narayana Bhat, Robert D. Preece, Charles A. Meegan (NSSTC)

Accreting pulsars are exceptional7y good 1aboratories for probing the detailed physics of accretion onto magnetic stars. While similar accretion flows also occur in other types of astrophysical systems, e.g. magnetic CVs, on $7 y$ neutron stars have a sma17 enough moment of inertia for the accretion of angular momentum to result in measurable changes in spin-frequency in a timescale of days. Long-term monitoring of accreting pulsar spin-frequencies and fluxes was demonstrated with the Burst and Transient Source Experiment (BATSE) on the Compton Gamma Ray observatory. Here we present sample results from BATSE, discuss measurement techniques appropriate for GBM, and estimate the expected GBM sensitivity. 


\section{Monitoring Accreting X-ray Pulsars with the GLAST Burst Monitor}

Colleen A. Wilson, M. H. Finger, S. K. Patel, P. N. Bhat, R. D. Preece, C. A. Meegan (NSSTC) 


\section{Outline}

- Introduction

- Examples of BATSE results

- Basic Technique

- Estimated sensitivity

- Summary 


\section{Accreting X-ray Pulsars}
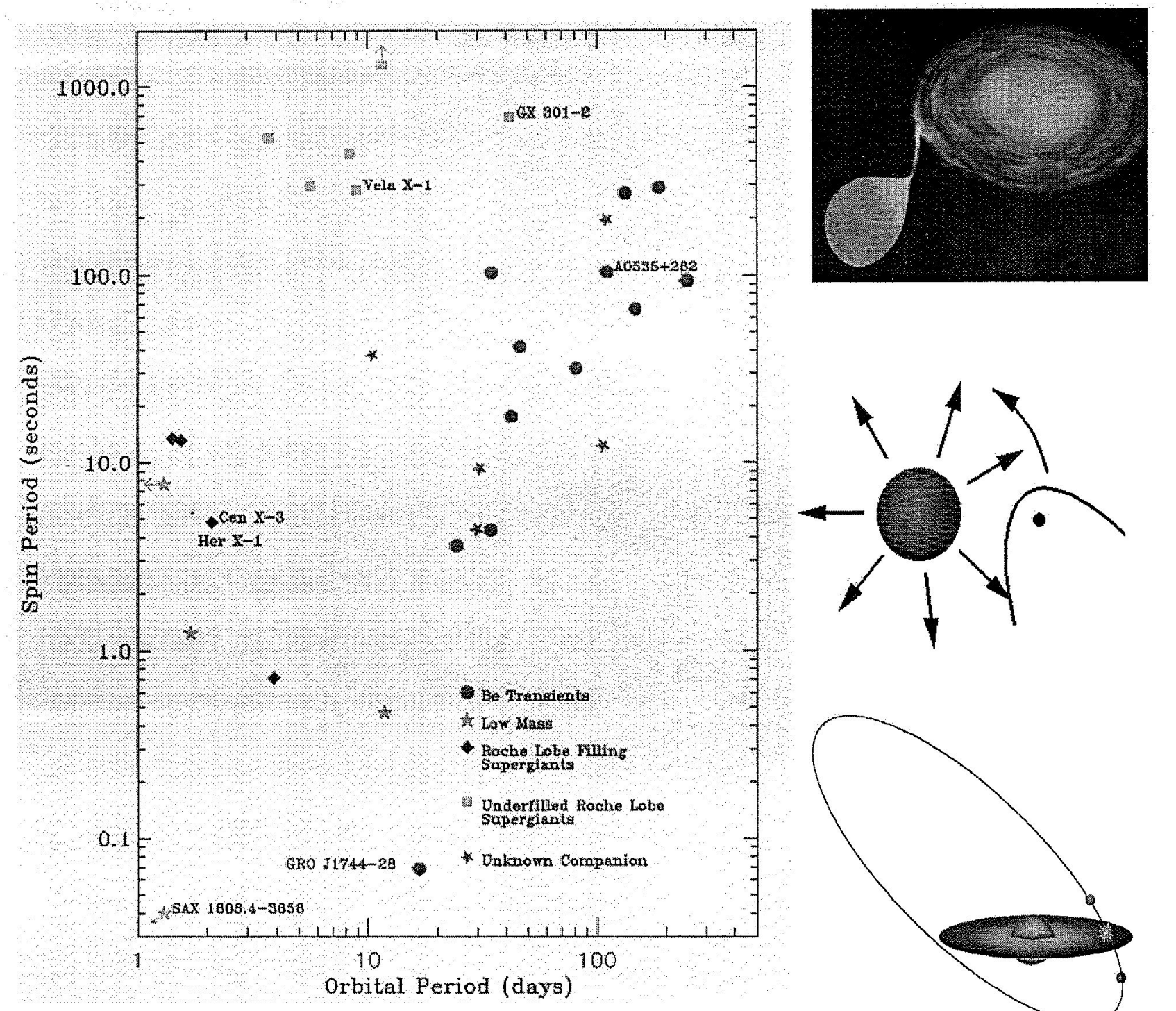

\section{Roche lobe overflow}

Wind accretion

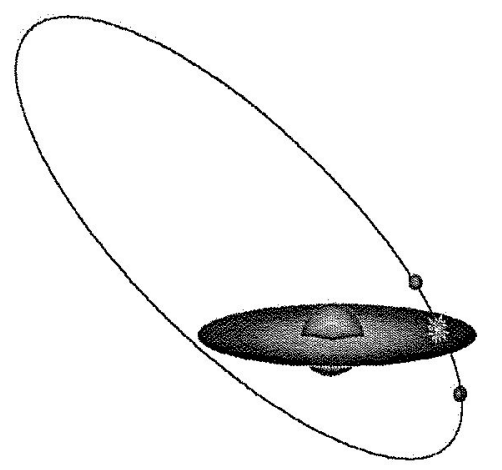

Be star's circumstellar disk 


\section{Vela X-1: a 283-s Wind-fed Pulsar}

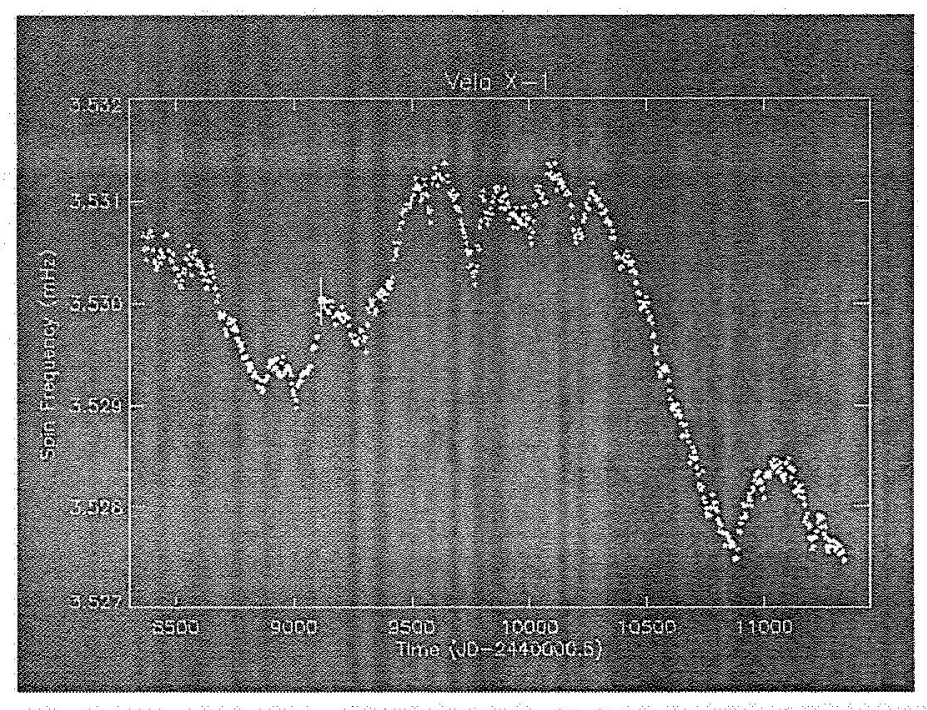

- Episodes of spin-up and spin-down with frequent reversals.

- Eclipses of the pulsar by its supergiant companion last about 2 days in its 8.96-day orbit.

- Large flares, like that observed with Integral in 2003, were also seen with BATSE 


\section{EXO 2030+375: a 42-s Pulsar in a Be/X-ray Binary}
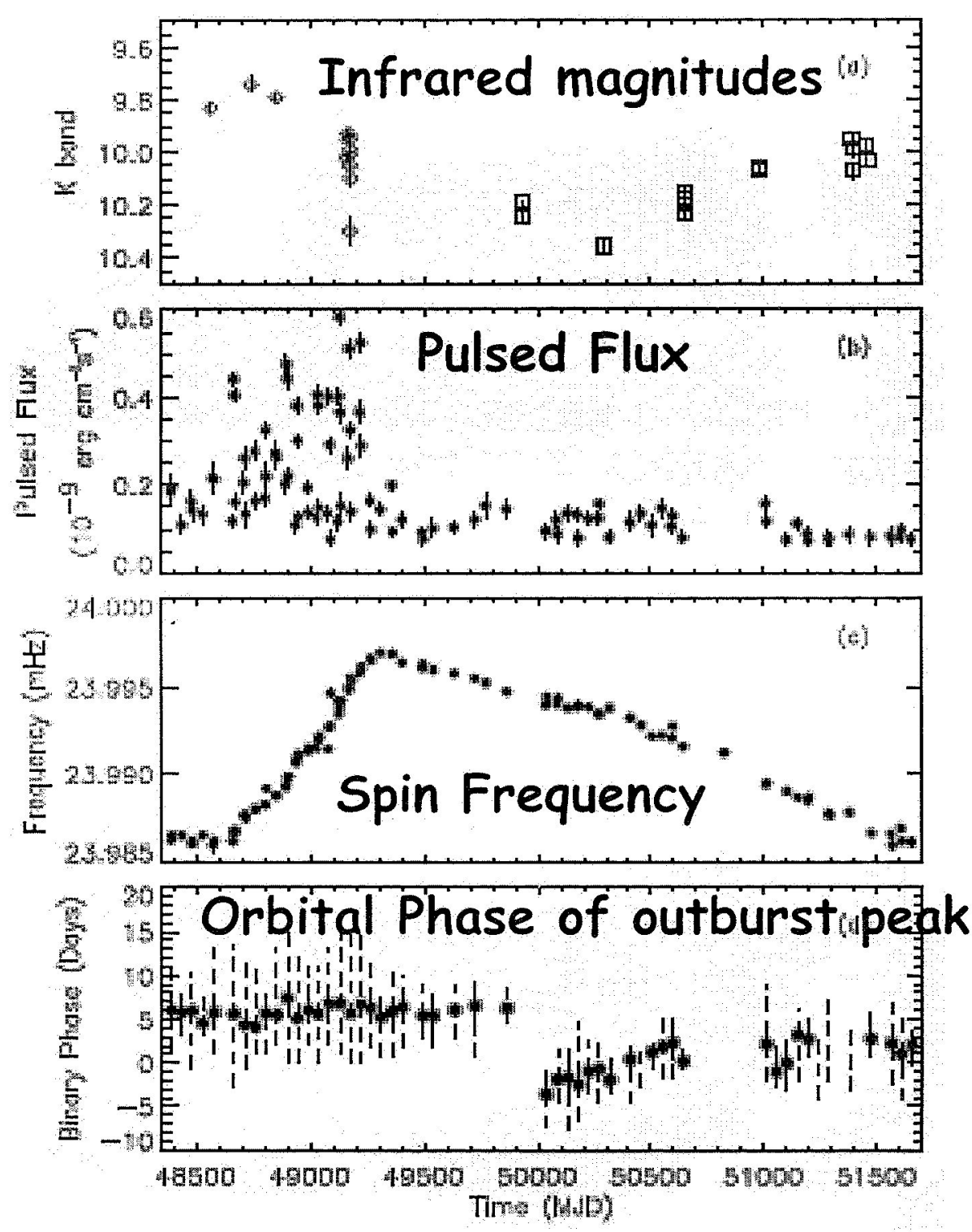

(Wilson et al. 2002, ApJ , 570, 287)
- There are two disks in this system: an excretion disk around Be star and accretion disk around pulsar.

- A decline in the density of the Be star's disk caused a drop in pulsed flux and $a$ transition from spin-up to spin-down.

- The orbital phase of the transient outbursts shifted in 1995, accompanied by a change in the $H \alpha$ profile, indicating the presence of a global one-armed oscillation in the Be disk. 


\section{Basic Technique}

- Use all data when pulsar of interest is above the horizon.

- Fit short segments ( 300 s) of data with a background model and a pulse profile model.

- Do a grid search in frequency offset from the original model, aligning and combining all profiles in a longer time interval, using a modified $Z^{2}$ test.

- Account for aperiodic noise using a fit to a mean pulse profile or a power spectrum. 


\section{Estimated GBM Pulsed Flux Sensitivity}

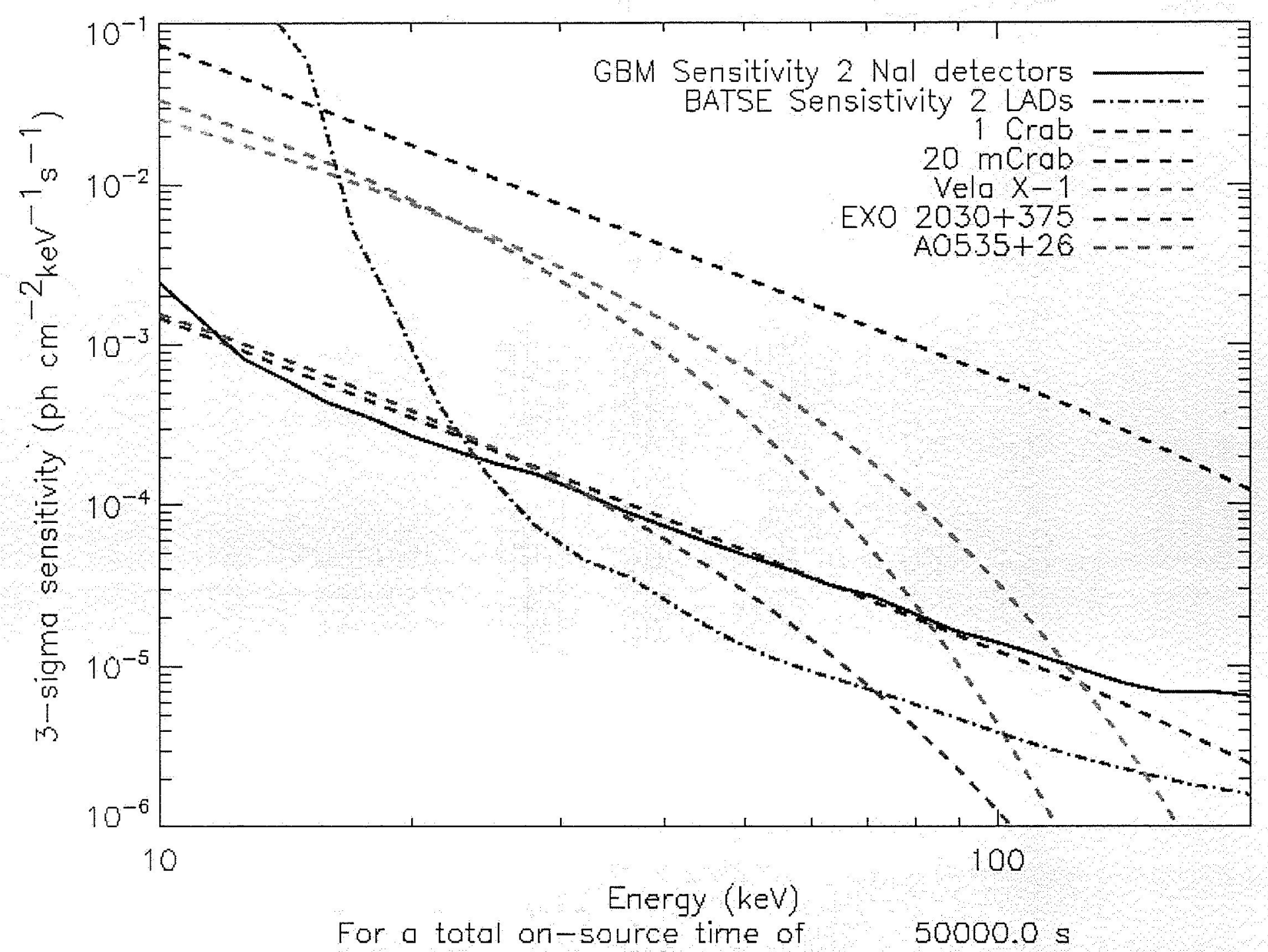




\section{Summary}

- We plan to propose a pulsar monitor, based upon the BATSE heritage, to measure spin frequency and pulsed flux with GBM.

- The fortunate combination of the GBM sensitivity below $25 \mathrm{keV}$ and the shape of typical pulsar spectra make this monitor able to detect the majority of sources detected with BATSE.

- This technique is much more sensitive than Earth Occultation because it uses all of the data when the source is above the horizon, but it is only suited for pulsed sources.

- The continuous data types provided by GBM are well suited to this task. 